\title{
The semantic Web: prospects $\&$ challenges
}

\section{Michael Wilson}

Manager, W3C Office in UK \& Ireland,

CCLRC Rutherford Appleton Laboratory 


\section{CCLRC Rutherford Appleton Laboratory}

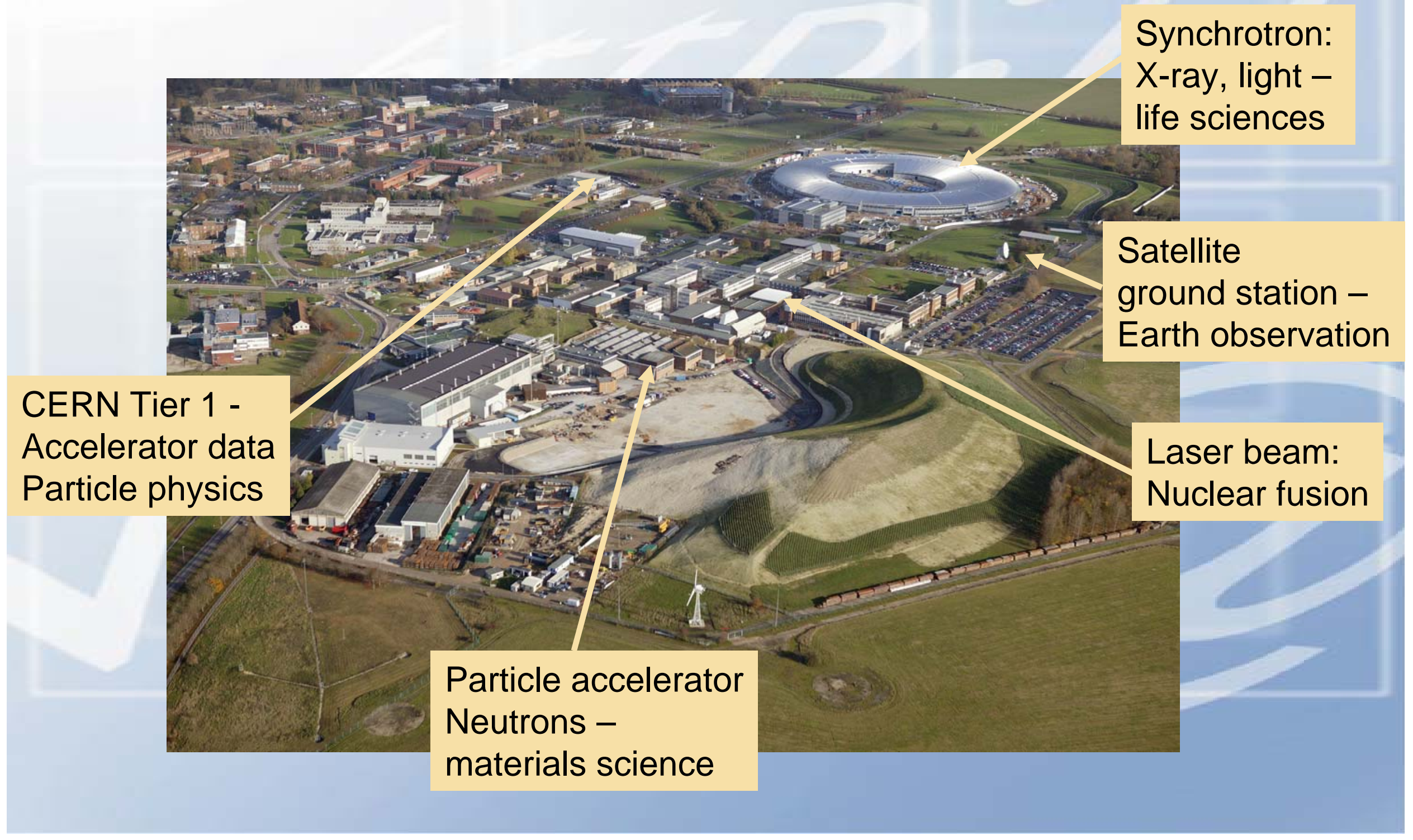




\section{W3C Web Architecture}

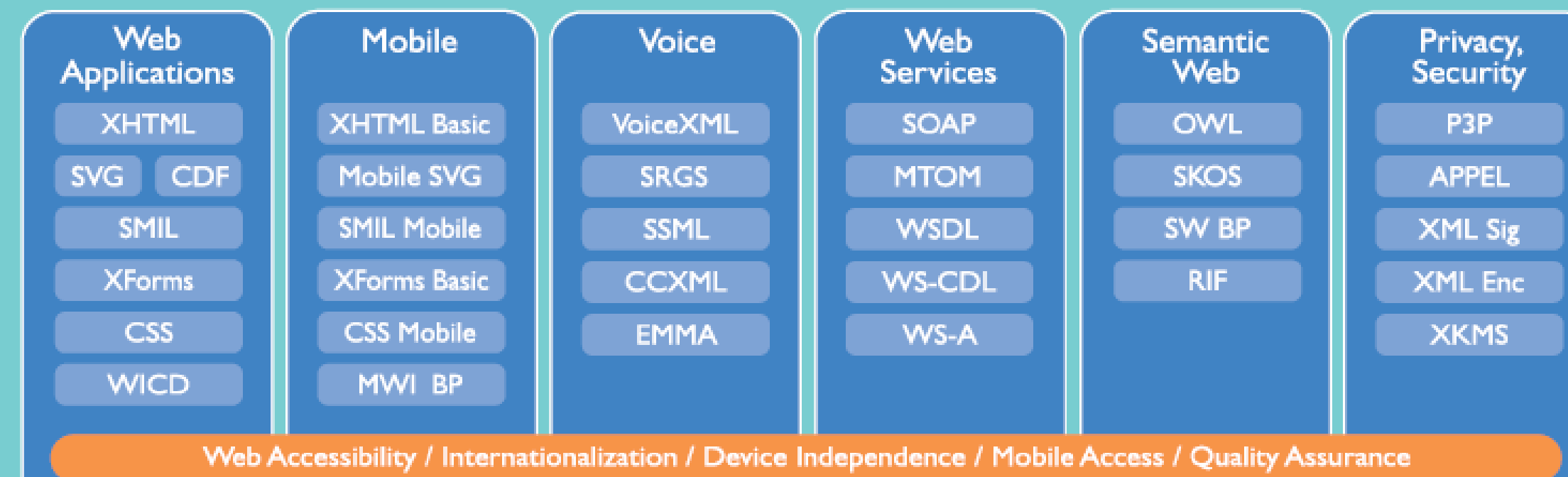

Web Accessibility / Internationalization / Device Independence / Mobile Access / Quality Assurance

XML, Namespaces, Schemas, XQuery/XPath, XSLT, DOM, XML Base, XPointer, RDF/XML, SPARQL

XML Infoset, RDF(S) Graph

Web Architectural Principles

URI/IRI, HTTP

One Web 


\section{Comparing Web and semantic Web development}

Web

- 1989 proposal

- +2 yrs portable browser

- +5 yrs commercial browser, index, crawler

- +6 yrs 73,500 servers

- +10 yrs 3\% US GDP from web
Semantic Web

- 1996 proposal

-+3yrs RDF agreed

-+8yrs browser, OWL, RDF Schema

-+9 yrs demonstration

applications

-+10 yrs commercial DB

support 


\section{The semantic Web}

- 1996 - metadata to describe the data on the Web.

- 1998 - query languages, inference rules \& proof validation

- 2001 - bring structure to the meaningful content of web pages, creating an environment where software agents roaming from page to page can readily carry out sophisticated tasks for users.

- 2006 -The "Semantic Web" is an infrastructure extending the current Web for the interchange and the integration of data on the Web. 


\section{Semantic Web Architecture \& Technologies - 2001}

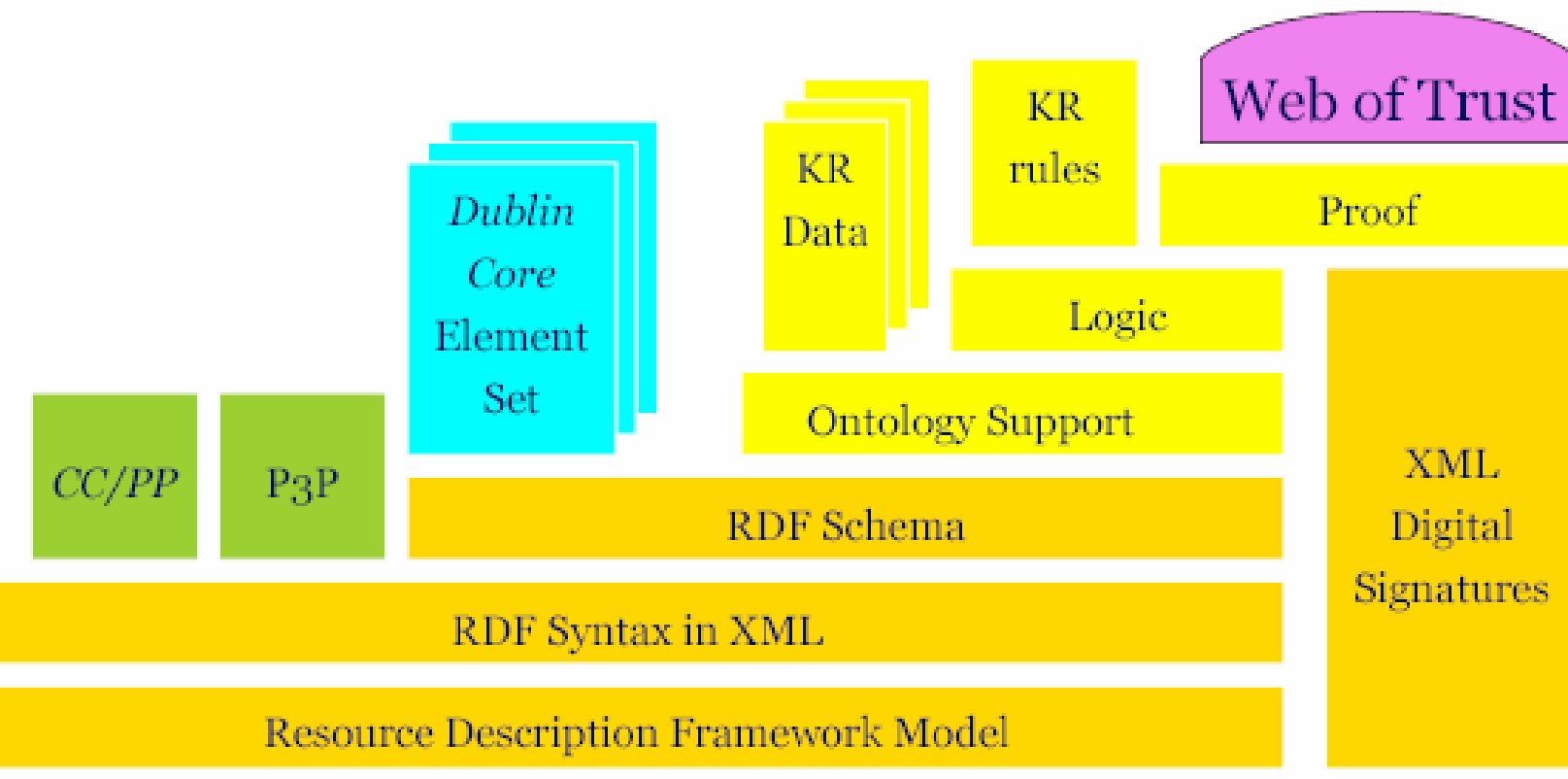

Resource Description Framework Model

XML: Structure Documents 


\section{Semantic Web Architecture \& Technologies - 2006}

- To make data machine processable, we need:

- unambiguous names for resources (that may also bind data to real world objects): URI

- a common data model to access, connect, describe the resources: RDF

- access to that data, join the web: SPARQL

- common access to XML and RDF: GRDDL

- define common vocabularies, ontologies: RDFS, OWL, SKOS

- Hyperlinks and the semantic Web - RDF/A

- Reasoning - Rule Interchange Format 


\section{GRDDL}

- Gleaning Resource Descriptions from Dialects of Languages ( GRDDL)

- mechanism to relate other XML syntaxes (especially XHTML dialects) to the RDF abstract syntax via transformations

- Example - HTML Snippet:

$<$ html $>$

$<$ head $><$ title $>$ Example of an HTML document $</$ title $>$

$</$ head $>$

Translates to RDF assertion:

$<$ document.html > dc:title "Example of an HTML document". - Further work in W3C to take GRDDL to a recommendation 


\section{SPARQL - RDF Query Language}

SELECT ?cat ?val \# note: not ?x!

WHERE \{ ?x rdf:value ?val. ?x category ?cat \}

Returns: [ ["Total Members",100], ["Total Members",200],..., ["Full Members", 10 ], ... ]

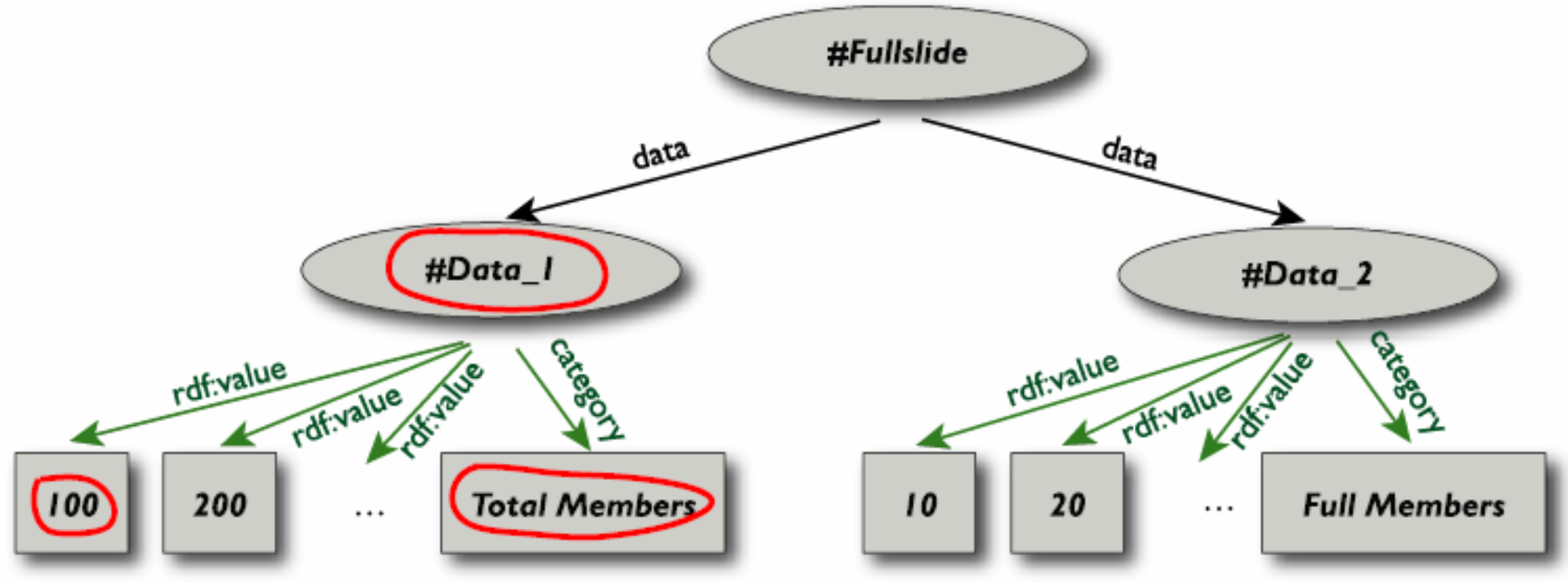

-Waiting on XQuery to go to recommendation before SPARQL can. 


\section{SPARQL - Application}

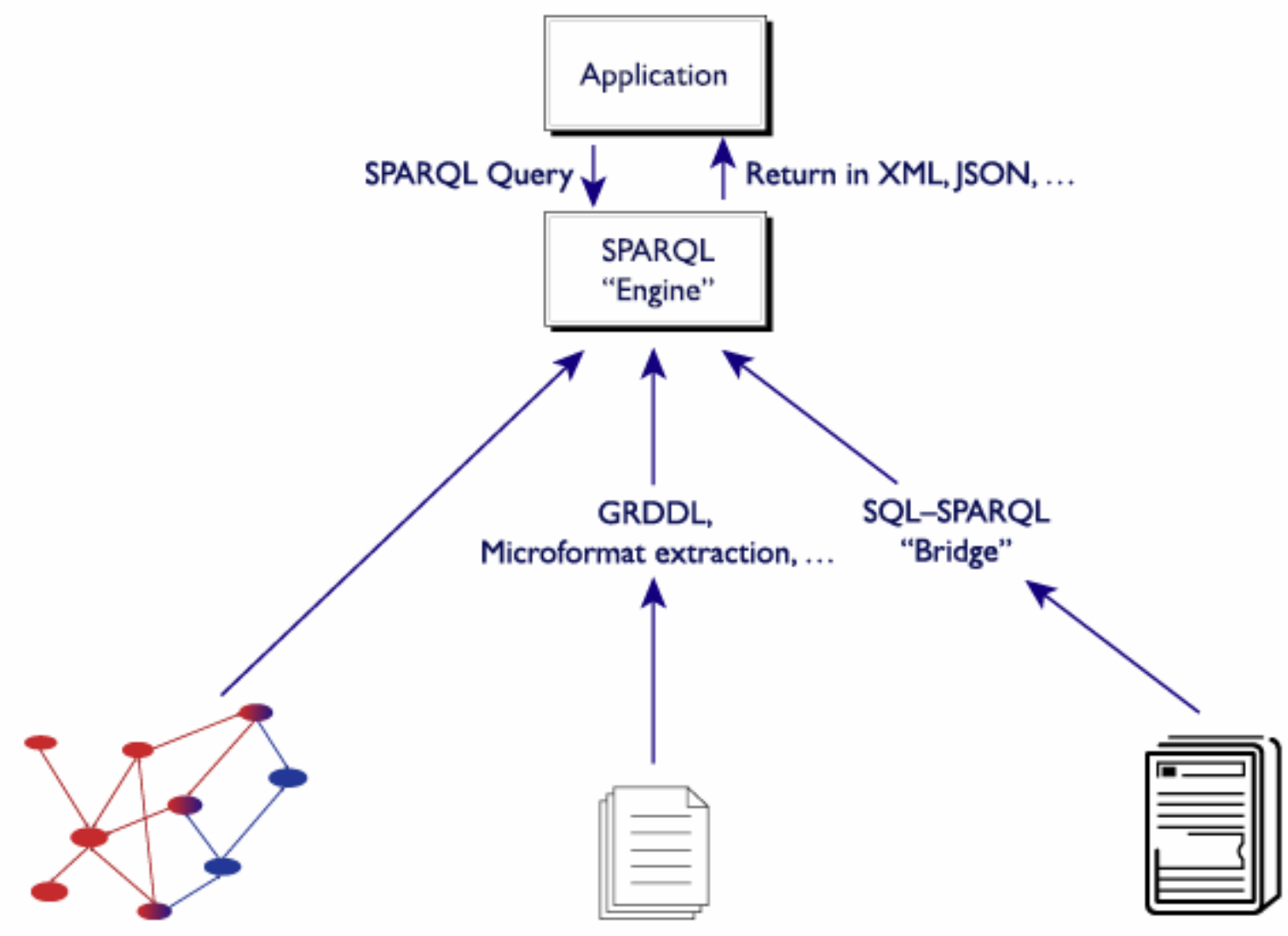

RDF Data

Documents (XHTML, XML, ...)

(Relational) Database 


\section{RDF-A}

- RDF/A is a set of attributes used to embed RDF in XHTML.

- Instead of:

If you want to contact me at work, you can either $<a$ href="mailto:jo.lambda@example.org">

email me</a $>$, or call +1 7778889999 .

Use:

If you want to contact me at work, you can either

$<$ a rel="foaf:mbox" href="mailto:jo.lambda@example.org"> email me</a>,

or call <span property="foaf: phone">+1 777888 $9999</$ span $>$.

To produce RDF triples:

foaf:mbox = "mailto:jo.lambda@example.org" foaf:phone $=$ "+1 777888 9999" 


\section{Layering of the semantic Web}

- Each layer should meet a new market need

- Each layer should build on previous ones

- Each layer should provide ROI by itself 


\section{Commercial RDF support}

- Oracle v10.2

- Native RDF store

- Converters / loaders for existing RDF data

- RDF Query - An RDF_MATCH function which can be used in SQL to find graph patterns in RDF (similar to SPARQL)

- Over 100 Partners Thoroughly Test and Support Oracle Database 10g Release 2 


\section{Small Applications}

Small apps in RDF provide ROI:

RSS/Podcast

Dublin Core

XFN: bloggers

Calendar

Geo URL

FOAF

Thesaurus

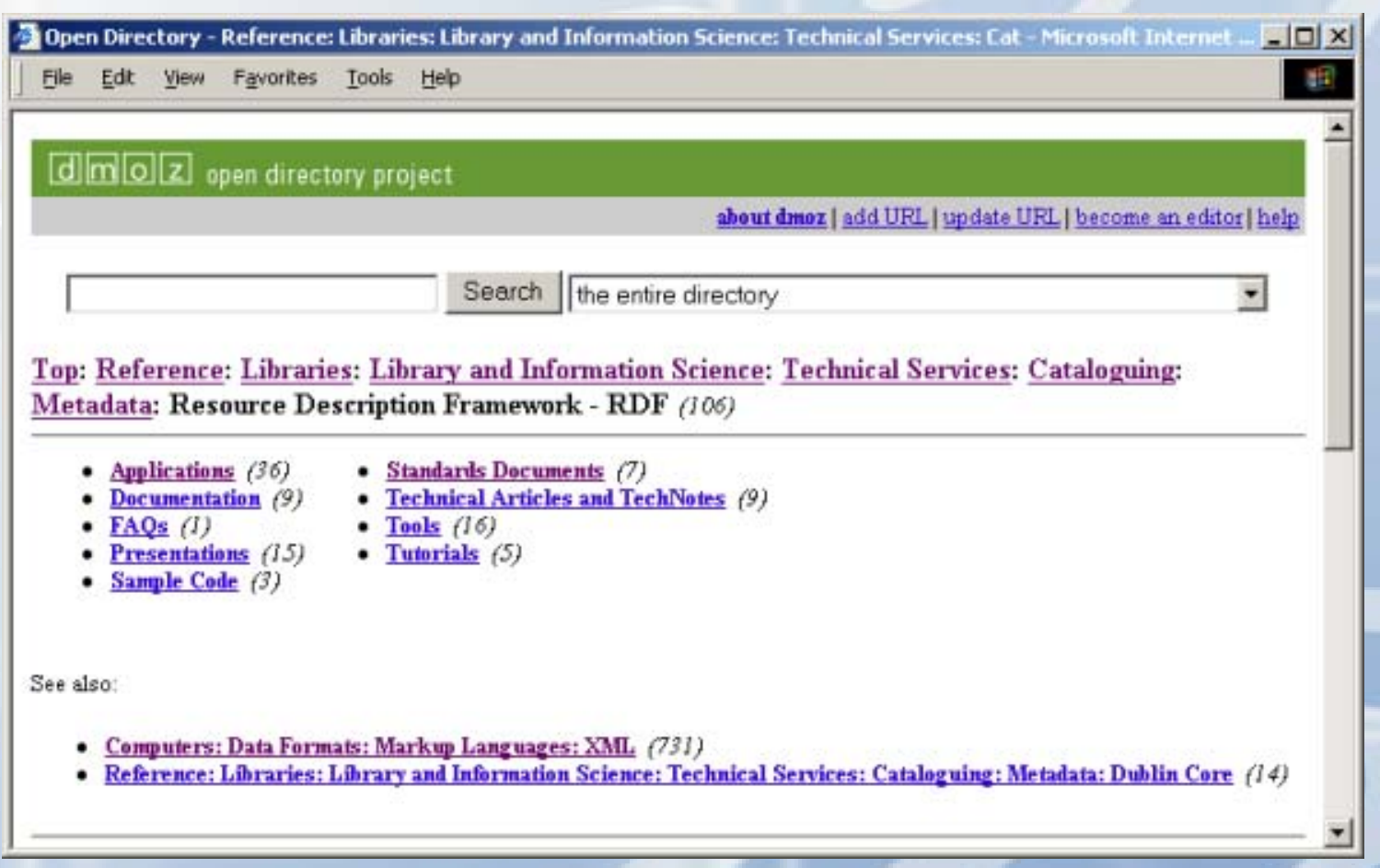

DMOZ is an open directory project represents the directory an its contents in RDF.

XML \& RDF 


\section{Applications: Data Integration}

- No "one schema" that can be used for describing everything

- No "right way" for describing / organizing anything

- Importance of "Partial Understanding"

- Things change, plan up front for it

- The value in "as needed" data integration

- Big wins come from many little ones

- The power of links - network effect

- Open-world, open solutions are cost effective 


\section{Application: data integration}

- Semantic integration of different data sources

- RDF/RDFS (possibly with OWL and/or SKOS) based vocabularies as an "interlingua" among system components

- Many different projects and R\&D on this: Boeing, MITRE Corp., Elsevier, EU Projects like Sculpteur and Artiste, national projects like MuseoSuomi. ...
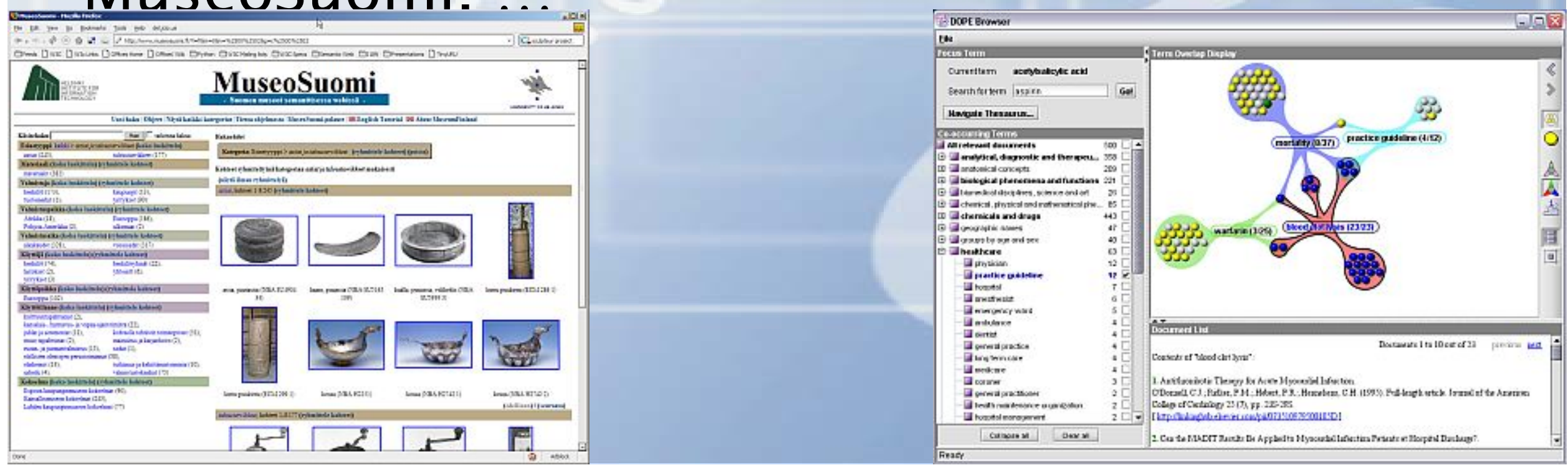


\section{Applications: Portals}

- Vodafone's Live Mobile Portal

- search application (e.g. ringtone, game, picture) using RDF

- page views per download decreased $50 \%$

- ringtone up 20\% in 2 months

- Sun's SwordFish: public queries for support, handbooks, etc, go through an internal RDF engine for White Paper Collections and System Handbook collections

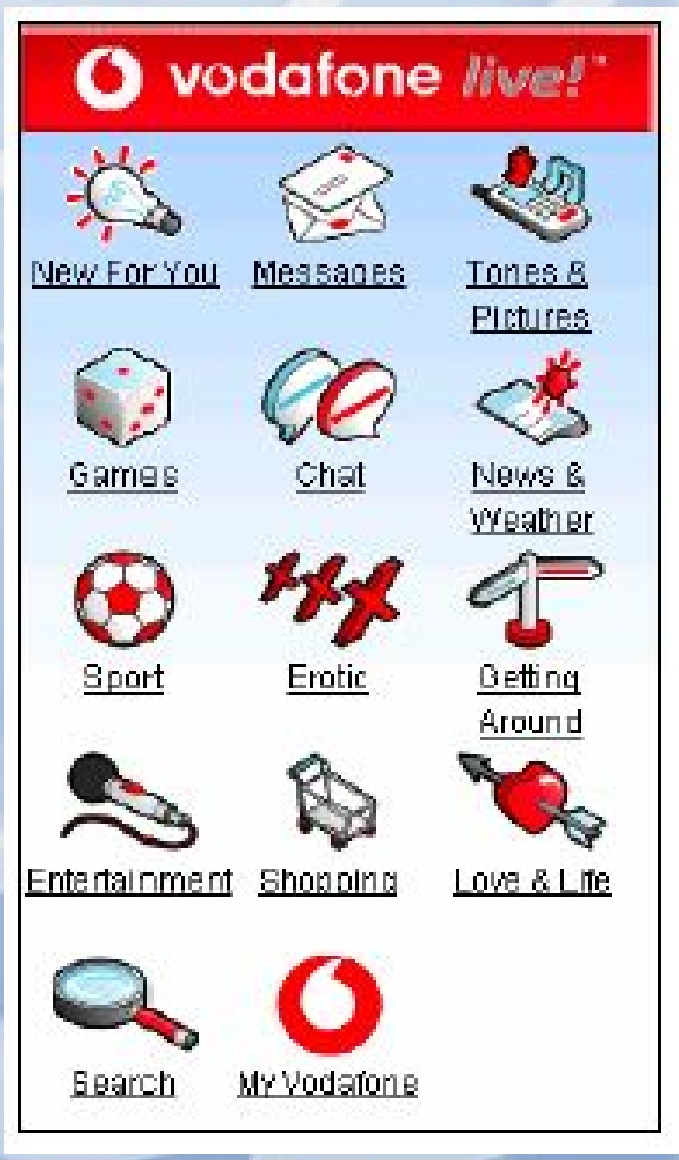

- Nokia has a somewhat similar support portal 


\section{Common Themes to successful SW applications}

- No "one schema" that can be used for describing everything

- No "right way" for describing / organizing anything

- Importance of "Partial Understanding"

- Things change, plan up front for it

- The value in "as needed" data integration

- Big wins come from many little ones

- The power of links - network effect

- Open-world, open solutions are cost effective 


\section{Summary}

- Evolution not the revolution of the Web

- Small parts added to the architecture

- Development and adoption are progressing and continuing

But ...

Research Challenges ... 


\section{Six research problems for the SW}

- Understanding ontological modelling

- Logical basis for inference

- Translating between ontologies

- Reasoning about agent's intentions

- Sociology of agents

- Governance of agents 


\section{Understanding ontological modelling}

- Guizzardi addressed some of these issues yesterday.

- OWL is an epistemological language for ontologies

- What is the development method for ontologies?

-What are the design languages ?

- What are the design trade-offs in ontology development?

- What is the ontological status of objects and relations? 


\section{Logical basis for inference}

- Are Description Logics insufficient for rule based reasoning?

- Are Horn clause logics required instead?

- The constraints on Horn clause logic and description logics are different.

- What are the consequences of using the two together? 


\section{Translating between ontologies}

- Today

- transform to common syntax

- Take union of concepts $\&$ axioms

- Define bridging axioms through an inference engine.

- Eventually

- Metaphors are assertions of conceptual identity

- Translation using metaphoric relations 


\section{Reasoning about agent's intentions}

- Certified Assertion based confidence

- Transfer/Generalisation of assertions ?

- Generalisation as trust - common intention

- How do we transfer/generalise intentions? 


\section{Sociology of agents}

- Agents operate in networks

- Agents cluster and herd

- What properties of the group behaviour of agents are important? 


\section{Governance of agents}

- Liability and Governance

- Extending

- the development method

- the run time system

- service quality 


\section{Conclusion}

- The larger vision of the SW is still valid

- It will take a long time to evolve from the web of today into a full SW

- Much research in more abstract areas is required before we will get there

- The opportunities are there for you to take 